\title{
The (Quasi-)Baerness of Skew Group Ring and Fixed Ring*
}

\author{
Hailan Jin ${ }^{1}$, Qinxue Zhao ${ }^{2}$ \\ Department of Mathematics, College of Sciences, Yanbian University, Yanji, China \\ E-mail: hljin98@ybu.edu.cn, hljin98@hanmail.net \\ Received July 15, 2011; revised September 1, 2011; accepted September 10, 2011
}

\begin{abstract}
In this paper, the (quasi-)Baerness of skew group ring and fixed ring is investigated. The following two results are obtained: if $R$ is a simple ring with identity and $G$ an outer automorphism group, then $R * G$ is a Baer ring; if $R$ is an Artinian simple ring with identity and $G$ an outer automorphism group, then $R^{G}$ is a Baer ring. Moreover, by decomposing Morita Context ring and Morita Context Theory, we provided several conditions of Morita Context ring, which is formed of skew group ring and fixed ring, to be (quasi-)Baer ring.
\end{abstract}

Keywords: Baer Ring, Quasi-Baer Ring, Morita Context Ring, Skew Group Ring, Fixed Ring, Simple Ring

\section{Introduction}

Throughout this paper all rings are associative with identity, all modules are unitary module. Recall that a ring $R$ is called (quasi-)Baer if the right annihilator of every nonempty subset (resp. every right ideal) of $R$ is generated by an idempotent as a right ideal [1]. These definitions are left-right symmetric. A ring $R$ is called right (resp. left) principally quasi-Baer ring (simply, right p.q.-Baer ring) if the right (resp. left) annihilator of every principally right (resp. left) ideal of $R$ is generated, as a right (resp. left) ideal, by an idempotent of $R$. A ring $R$ is called p.q.-Baer if $R$ is both right and left p.q.-Baer [1]. Baer ring is a quasi-Baer ring. Conversely, it may not hold. Baer ring and quasi-Baer ring are right p.q.-Baer ring, but converse may not true. In [2], properties of the skew group ring $A * G$ and the fixed ring $A^{G}$ of a $C^{*}$-Algebra $A$ with a group $G$ of ring automorphisms of $A$ has been investigated. By [1], we know that if $R$ is a semiprime p.q.-Baer ring with a finite group $G$ of X-outer ring automorphisms of $R$, then $R * G$ is a p.q.-Baer ring. Also in [1] example of a semiprime Baer ring $R$ with $G$ a finite group of X-outer ring automorphisms of $R$ such that $R$ has no nonzero $|G|$-torsion, but $R * G$ is not a Baer ring was provided.

In [3], Morita K introduced Morita Context. An alge-

${ }^{*}$ Foundation item: Projective Supported by Provincial Education Department of JiLin Eleventh-Five Year Science and Technology Research Program [2010] (Grant: No. 272). braic structure $(R, V, W, S, \psi, \varphi)$ [4] is called Morita Context if $R$ and $S$ are rings, $V={ }_{R} V_{S}$ is a $R-S$ bimodule, $W={ }_{S} W_{R}$ is a $S-R$ bimodule, $\psi: V \otimes_{S} W \rightarrow R$ is a $R$-bimodule middle $S$-linear homomorphism, $\varphi: \mathrm{W} \otimes_{\mathrm{R}} \mathrm{V} \rightarrow \mathrm{S}$ is a $S$-bimodule middle $R$-linear homomorphism with

$\psi(\mathrm{v} \otimes \mathrm{w}) \mathrm{v}^{\prime}=\mathrm{v} \varphi\left(\mathrm{w} \otimes \mathrm{v}^{\prime}\right), \quad \varphi(\mathrm{w} \otimes \mathrm{v}) \mathrm{w}^{\prime}=\mathrm{w} \psi\left(\mathrm{v} \otimes \mathrm{w}^{\prime}\right)$, for any $\mathrm{v}, \mathrm{v}^{\prime} \in \mathrm{V}$. Let

$C=\left(\begin{array}{cc}R & V \\ W & S\end{array}\right)=\left\{\left(\begin{array}{ll}a & v \\ w & b\end{array}\right) \mid a \in R, b \in S, v \in V, w \in W\right\}$.

Then $C$ is a ring under the addition of matrix and multiplication given by

$\left(\begin{array}{ll}a & v \\ w & b\end{array}\right)\left(\begin{array}{cc}a^{\prime} & v^{\prime} \\ w^{\prime} & b^{\prime}\end{array}\right)=\left(\begin{array}{cc}a a^{\prime}+\psi\left(v \otimes w^{\prime}\right) & a v^{\prime}+v b^{\prime} \\ w a^{\prime}+b w^{\prime} & \varphi\left(w \otimes v^{\prime}\right)+b b^{\prime}\end{array}\right)$.

This generalized matrix ring is called Morita Context ring [4]. In [4,5], properties of Morita Context ring with two module zero homomorphisms were investigated. Let $R$ be a ring with identity and $G$ a group. Then

$\left(\begin{array}{cc}R * G & R^{*} \\ R & R^{G}\end{array}\right)$ is a Morita Context ring. A module $M_{R}$ is called (resp. quasi-Baer) Baer module if for any subset (resp. submodule) $N$ of $M, r_{R}(N)=f R$ where $f^{2}=f \in R$. In [6], a ring $R$ is a Baer (resp. quasiBaer) if and only if $R_{R}$ is a Baer (resp. quasi-Baer) module.

This paper firstly provides counterexamples to prove that $R$ is a Baer ring, but skew group ring $R * G$ and 
fixed ring $R^{G}$ may not hold Baer ring. Also the conditions of skew group ring and fixed ring to be (quasi-) Baer ring are investigated. Moreover, by decomposing Morita Context ring as finite direct sum of Baer modules and Morita Context Theory, we obtain the ways to judge the (quasi-)Baerness of a Morita Context ring, which is formed of skew group ring and fixed ring.

\section{Preliminary}

Definition 2.1 Let $R$ be a ring and $G$ a group, $R * G=\left\{\sum_{g \in G} r_{g} g \mid r_{g} \in R, r_{g} \neq 0\right.$ for finite many $\left.g \in G\right\}$.

Defined addition and multiplication in $R * G$ as follows: for any $\sum_{g \in G} r_{g} g, \sum_{g \in G} r_{g}^{\prime} g$, and $\sum_{h \in G} r_{h} h \in R * G$, $\sum_{g \in G} r_{g} g+\sum_{g \in G} r_{g}^{\prime} g=\sum_{g \in G}\left(r_{g}+r_{g}^{\prime}\right) g$, $\left(\sum_{g \in G} r_{g} g\right)\left(\sum_{h \in G} r_{h} h\right)=\sum_{g \in G} \sum_{h \in G} r_{g} r_{h}^{g^{-1}} g h$,

Then $R * G$ is a ring which is called skew group ring.

There exists a ring $R$, which is a Baer ring, but $R * G$ is not a Baer ring.

Example 2.1 Let $R=\left(\begin{array}{cc}F & F \\ 0 & F\end{array}\right)$ with a field $F$ of characteristic 2. Then $R$ is a Baer ring. For any

$\left(\begin{array}{ll}a & b \\ 0 & c\end{array}\right) \in R$, define $g \in \operatorname{Aut}(R)$ by $g\left(\left(\begin{array}{ll}a & b \\ 0 & c\end{array}\right)\right)=\left(\begin{array}{ll}1 & 1 \\ 0 & 1\end{array}\right)^{-1}\left(\begin{array}{ll}a & b \\ 0 & c\end{array}\right)\left(\begin{array}{ll}1 & 1 \\ 0 & 1\end{array}\right)$. Then $g^{2}=1$

since field $F$ of characteristic 2 . Let $G=\{1, g\}$. Then $R * G$ is not a quasi-Baer ring. So it is not a Baer ring.

In fact, for the principally right ideal $(1+g)(R * G)$ of $R * G$,

$$
\begin{aligned}
& r_{R * G}((1+g)(R * G)) \\
& =\left\{\left(\begin{array}{cc}
x & y \\
0 & 0
\end{array}\right)+\left(\begin{array}{cc}
x & x+y \\
0 & 0
\end{array}\right) g \mid x, y \in F\right\} .
\end{aligned}
$$

Suppose that $r_{R * G}((1+g)(R * G))=e(R * G)$ for some $e^{2}=e \in R * G$. Note that the idempotents of $R * G$ are $0,1,\left(\begin{array}{ll}1 & a \\ 0 & 0\end{array}\right)+\left(\begin{array}{ll}0 & b \\ 0 & 0\end{array}\right) g$ and $\left(\begin{array}{ll}0 & a \\ 0 & 1\end{array}\right)+\left(\begin{array}{ll}0 & b \\ 0 & 0\end{array}\right) g$ with $a, b \in F$. Thus $e \in r_{R * G}((1+g)(R * G))$. So the only choice for $e$ is 0 . If $R * G$ is a quasi-Baer ring, then $r_{R * G}((1+g)(R * G))=0$, which is a contradiction. Therefore $R * G$ is not a Baer ring.

A ring $R$ is called simple ring if $R^{2} \neq 0$ and $R$ has no proper ideal. A right module $M$ over a ring $R$ is called simple provided $M R \neq 0$ and $M$ has no proper submodules.

A ring $R$ is said to be a semisimple if the intersection $J(R)$ of all the maximal right ideals of $R$ is 0 . A nonzero module $M_{R}$ over a ring $R$ is called semisimple if $M$ is the direct sum of a family of simple modules.

Simple ring with identity is a semisimple ring [7].

Definition 2.2 [8] An automorphism $g$ of a ring $R$ is said to be inner if there exists a unit $u \in U(R)$ such that $g(x)=u x u^{-1}$, for all $x \in R$.

A subgroup $G$ of $\operatorname{Aut}(R)$ is said to be an outer automorphism group if identity mapping is the only inner automorphism in $G$.

Lemma 2.1 [8] Let $R$ be a simple ring with identity and $G$ an outer automorphism group. Then $R * G$ is a simple ring; If $R$ is an Artinian ring, then $R * G$ is an Artinian simple ring.

Semisimple ring $R$ is a left Artinian ring if and only if it is a right Artinian ring [7].

Lemma 2.2 [7] A module $M$ over a right Artinian semisimple ring $R$ with identity is a semisimple.

Lemma 2.3 [9] Semisimple ring is a Baer ring.

Also Semisimple module is a Baer module [10].

Definition 2.3 Let $R$ be a ring and $G$ a group, $\delta: G \rightarrow \operatorname{Aut}(R)$ a homomorphism of group. Then $R^{G}=\left\{r \in R \mid r^{g}=r, \forall g \in G\right\}$ is said to be a fixed ring of $R$ under $G$ where $r^{g}=(\delta(g))(r)$.

Example 2.2 Let $R$ be a ring, $G=\left\{f \in \operatorname{Aut}(R) \mid f(r)=\operatorname{ara}^{-1}, \forall r \in R, a \in U(R)\right\} \quad$ and $\delta=\left.i d\right|_{G}$. Then

$$
\begin{aligned}
R^{G} & =\left\{r \in R \mid r^{g}=r, \forall g \in G\right\} \\
& =\left\{r \in R \mid a r a^{-1}=r, a \in U(R)\right\} \\
& =\{r \in R \mid a r=r a, \forall a \in U(R)\},
\end{aligned}
$$

i.e., $R^{G}$ is the set of all the elements, which are commutative with the units of $R$.

Lemma 2.4 [8] Let $R$ be a ring with identity and $G$ a group. Then $R^{G} \cong \operatorname{End}\left(R_{R * G}\right)$.

Lemma 2.5 [9] Let $R$ be a ring with identity and $M_{R}$ a semisimple right module. Then $\operatorname{End}\left(M_{R}\right)$ is a Baer ring.

\section{Main Results}

Theorem 3.1 Let $R$ be a simple ring with identity and $G$ an outer automorphism group. Then $R * G$ is a Baer ring.

Proof. It is evident from lemma 2.1 and lemma 2.2.

Definition 3.1 [1] Let $R$ be a semiprime ring. For 
$g \in \operatorname{Aut}(R)$, let $\phi_{g}=\left\{x \in Q^{r}(R) \mid x r^{g}=r x, \forall r \in R\right\}$. Then automorphism $g$ of ring $R$ is called $X$-outer if $\phi_{g}=0$. A subgroup $G$ of $\operatorname{Aut}(R)$ is called $X$-outer if every $1 \neq g \in G$ is a $X$-outer.

Theorem 3.2 Let $R$ be a semiprime ring and $G$ a $\mathrm{X}$-outer automorphism group. If $R$ is a simple ring with identity, then $R * G$ is a Baer ring.

Proof. Simple ring with identity is a semisimple ring. It is evident from lemma 2.2.

Example 3.1 There exists a Baer ring $R$ with $R^{G}$ is not Baer ring. Let $R$ be a ring defined in example 2.1. Then $U(R)=\left\{\left(\begin{array}{ll}1 & 0 \\ 0 & 1\end{array}\right),\left(\begin{array}{ll}1 & 1 \\ 0 & 1\end{array}\right)\right\}$. For $\left(\begin{array}{ll}a & b \\ 0 & c\end{array}\right) \in R$, if $\left(\begin{array}{ll}a & b \\ 0 & c\end{array}\right)\left(\begin{array}{ll}1 & 1 \\ 0 & 1\end{array}\right)=\left(\begin{array}{ll}1 & 1 \\ 0 & 1\end{array}\right)\left(\begin{array}{ll}a & b \\ 0 & c\end{array}\right)$, then $a=c$. Thus $R^{G}=\left\{\left(\begin{array}{ll}0 & 0 \\ 0 & 0\end{array}\right),\left(\begin{array}{ll}1 & 0 \\ 0 & 1\end{array}\right),\left(\begin{array}{ll}1 & 1 \\ 0 & 1\end{array}\right),\left(\begin{array}{ll}0 & 1 \\ 0 & 0\end{array}\right)\right\}$ by example 2.2. All the idempotents of $R^{G}$ are $\left(\begin{array}{ll}0 & 0 \\ 0 & 0\end{array}\right)$ and $\left(\begin{array}{ll}1 & 0 \\ 0 & 1\end{array}\right)$. However, for $\left(\begin{array}{ll}0 & 1 \\ 0 & 0\end{array}\right) \in R^{G}$, $r_{R^{G}}\left(\left(\begin{array}{ll}0 & 1 \\ 0 & 0\end{array}\right)\right)=\left\{\left(\begin{array}{ll}0 & 0 \\ 0 & 0\end{array}\right),\left(\begin{array}{ll}0 & 1 \\ 0 & 0\end{array}\right)\right\} \neq e R^{G}$, where $e^{2}=e \in R^{G}$. Therefore, $R^{G}$ is not a Baer ring.

Theorem 3.3 Let $R$ be a simple ring with identity and $G$ an outer automorphism group. If $R$ is an Artinian ring, then $R^{G}$ is a Baer ring.

Proof. By lemma $2.1 R * G$ is a simple ring since $R$ is a simple ring with identity and $G$ an outer automorphism group. If $R$ is an Artinian ring, then $R * G$ is an Artinian simple ring with identity. Consequently, $R * G$ is an Artinian semisimple ring. By lemma 2.4, $R_{R * G}$ is a semisimple module. So $\operatorname{End}\left(R_{R * G}\right)$ is a Baer ring by lemma 2.5. Moreover, $R^{G}$ is a Baer ring by lemma 2.3 .

Theorem 3.4 Let $C=\left(\begin{array}{cc}R & V \\ W & S\end{array}\right)$ be a Morita Context ring. If $R$ and $S$ are right Artinian simple ring, then $C$ is a Baer ring.

Proof. For any $\left(\begin{array}{cc}r & v \\ w & s\end{array}\right) \in\left(\begin{array}{cc}R & V \\ W & S\end{array}\right),\left(\begin{array}{cc}r_{0} & v_{0} \\ 0 & 0\end{array}\right)$, $\left(\begin{array}{cc}r_{1} & v_{1} \\ 0 & 0\end{array}\right) \in\left(\begin{array}{cc}R & V \\ 0 & 0\end{array}\right)$, we have $\left(\begin{array}{cc}r_{0} & v_{0} \\ 0 & 0\end{array}\right)-\left(\begin{array}{cc}r_{1} & v_{1} \\ 0 & 0\end{array}\right) \in\left(\begin{array}{cc}R & V \\ 0 & 0\end{array}\right)$, and

$$
\begin{aligned}
& \left(\begin{array}{cc}
r_{0} & v_{0} \\
0 & 0
\end{array}\right)\left(\begin{array}{cc}
r & v \\
w & s
\end{array}\right) \\
& =\left(\begin{array}{cc}
r_{0} r+\psi\left(v_{0} \otimes w\right) & r_{0} v+v_{0} s \\
0 & 0
\end{array}\right) \in\left(\begin{array}{ll}
R & V \\
0 & 0
\end{array}\right) .
\end{aligned}
$$

So $\left(\begin{array}{cc}R & V \\ 0 & 0\end{array}\right)$ is a right ideal of $C$. Similarly, $\left(\begin{array}{cc}0 & 0 \\ W & S\end{array}\right)$ is a right ideal of $C$. Because for any

$\left(\begin{array}{ll}r & v \\ w & s\end{array}\right) \in\left(\begin{array}{cc}R & V \\ W & S\end{array}\right)$,

$\left(\begin{array}{cc}r & v \\ w & s\end{array}\right)=\left(\begin{array}{cc}r & v \\ 0 & 0\end{array}\right)+\left(\begin{array}{cc}0 & 0 \\ w & s\end{array}\right) \in\left(\begin{array}{cc}R & V \\ 0 & 0\end{array}\right)+\left(\begin{array}{cc}0 & 0 \\ W & S\end{array}\right)$, and $\left(\begin{array}{cc}R & V \\ 0 & 0\end{array}\right) \cap\left(\begin{array}{cc}0 & 0 \\ W & S\end{array}\right)=0$. Thus $\left(\begin{array}{cc}R & V \\ W & S\end{array}\right) \cong\left(\begin{array}{cc}R & V \\ 0 & 0\end{array}\right) \oplus\left(\begin{array}{cc}0 & 0 \\ W & S\end{array}\right) . \quad R$ is the direct sum of minimal right ideals $J_{k}$ since $R$ is a right Artinian simple ring, where $k=1, \cdots, m, m \in \mathbb{Z}$. Since $\left(\begin{array}{cc}J_{k} & J_{k} V \\ 0 & 0\end{array}\right)$ is a minimal right ideal of $C$, it follows that $\left(\begin{array}{ll}R & V \\ 0 & 0\end{array}\right)$ is the direct sum of simple module. It is similar to $\left(\begin{array}{cc}0 & 0 \\ W & S\end{array}\right)$. Thus $C=\left(\begin{array}{cc}R & V \\ W & S\end{array}\right)$ is the direct sum of several simple module. Consequently, $\left(\begin{array}{cc}R & V \\ W & S\end{array}\right)$ is a semisimple module. Since semisimple module is a Baer module and $C$ a Baer module. Therefore, it is a Baer ring.

Baer ring is a quasi-Baer ring. Accordingly, if $R_{R}$ and $S_{S}$ are simple module, then $C$ is a quasi-Baer ring.

Example 3.2 Let $R$ be a ring with identity and $G$ a group. Obviously, $R$ is a $R^{G}-R * G$ bimodule. Let $R^{*}=\operatorname{Hom}\left(R_{R * G}, R * G_{R * G}\right)$. Then $R^{*}$ is a $R * G-R^{G}$ bimodule. For any $r, s \in R, \alpha, \beta \in R^{*}$, define:

$\psi: R^{*} \otimes_{R^{G}} R \rightarrow R * G, \quad \beta \otimes s \rightarrow \beta \cdot s=\beta ;$

$\varphi: R \otimes_{R * G} R^{*} \rightarrow R^{G}, \quad r \otimes \alpha \rightarrow(r \cdot \alpha)\left(1_{R}\right)=r \cdot\left(\alpha\left(1_{R}\right)\right)$.

Then $\left(\begin{array}{cc}R * G & R^{*} \\ R & R^{G}\end{array}\right)$ is a Morita Context ring. By lemma 2.1 if $R$ is an Artinian simple ring with identity and $G$ an outer automorphism group, then $R * G$ and $R^{G}$ are simple ring. By theorem $3.4\left(\begin{array}{cc}R * G & R^{*} \\ R & R^{G}\end{array}\right)$ is a Baer 
ring. Thus it is a quasi-Baer ring.

Theorem 3.5 Let $C=\left(\begin{array}{cc}R & V \\ W & S\end{array}\right)$ be a semisimple Artinian ring. Then $R$ and $S$ are quasi-Baer ring.

Proof. Since $C=\left(\begin{array}{cc}R & V \\ W & S\end{array}\right)$ is a semisimple Artinian ring, it follows that every right ideal of $C$ is of the form $e C$. Thus for any nonzero right ideal $I$ of $R$, there exists $\left(\begin{array}{cc}r_{1} & v_{1} \\ w_{1} & s_{1}\end{array}\right)^{2}=\left(\begin{array}{cc}r_{1} & v_{1} \\ w_{1} & s_{1}\end{array}\right) \in\left(\begin{array}{cc}R & V \\ W & S\end{array}\right)$ such that $\left(\begin{array}{cc}r_{R}(I) & V \\ 0 & 0\end{array}\right)=\left(\begin{array}{cc}r_{1} & v_{1} \\ W_{1} & S_{1}\end{array}\right)\left(\begin{array}{cc}R & V \\ W & S\end{array}\right)$. Accordingly, $r_{R}(I)=r_{1} R+\psi\left(v_{1} \otimes W\right)$. In fact, for $\forall r_{1} r+\psi\left(v_{1} \otimes w_{1}\right)$, $r_{1} s+\psi\left(v_{1} \otimes w_{2}\right) \in r_{1} R+\psi\left(v_{1} \otimes W\right)$, we have

$$
\begin{aligned}
& r_{1} r+\varphi\left(v_{1} \otimes w_{1}\right)-\left(r_{1} s+\varphi\left(v_{1} \otimes w_{2}\right)\right) \\
& =r_{1}(r-s)+\psi\left(v_{1} \otimes\left(w_{1}-w_{2}\right)\right) \in r_{1} R+\psi\left(v_{1} \otimes W\right),
\end{aligned}
$$

and

$$
\begin{aligned}
& \left(r_{1} r+\psi\left(v_{1} \otimes w_{1}\right)\right) r^{\prime} \\
& =r_{1} r r^{\prime}+\psi\left(v_{1} \otimes\left(w_{1} r^{\prime}\right)\right) \in r_{1} R+\psi\left(v_{1} \otimes W\right)
\end{aligned}
$$

So $r_{R}(I)=r_{1} R+\psi\left(v_{1} \otimes W\right)$ is a right ideal of $R$. By $\left(\begin{array}{cc}r_{R}(I) & V \\ 0 & 0\end{array}\right)=\left(\begin{array}{cc}r_{1} & v_{1} \\ w_{1} & s_{1}\end{array}\right)\left(\begin{array}{cc}R & V \\ W & S\end{array}\right)$ we have $w_{1} R+s_{1} W=0, \quad w_{1} V+s_{1} S=0$. Thus $w_{1}=s_{1}=0$. Since $\left(\begin{array}{ll}r_{1} & v_{1} \\ w_{1} & S_{1}\end{array}\right)$ is an idempotent of $R$, it follows that $r_{1}^{2}=r_{1}, \quad r_{1} v_{1}=v_{1}$. Accordingly,

$$
\begin{aligned}
r_{R}(I) & =r_{1} R+\psi\left(v_{1} \otimes W\right)=r_{1} R+\psi\left(r_{1} v_{1} \otimes W\right) \\
& =r_{1} R+r_{1} \psi\left(v_{1} \otimes W\right)=r_{1} R,
\end{aligned}
$$

i.e., $r_{R}(I)$ is a right ideal of $R$ generated by an idempotent. Therefore $R$ is a quasi-Baer ring. Similarly, $S$ is a quasi-Baer ring.

\section{References}

[1] H. L. Jin, "Principally Quasi-Baer Skew Group Rings and Fixed Rings,” Sc.D. Dissertation, College of Science, Pusan National University, Pusan, 2003.

[2] P. Ara and M. Mathieu, "Local Multipliers of $C^{*}$-Algebras," Springer, Berlin-Heidelberg, New York, 2003.

[3] K. Morita, "Duality for Modules and Its Application to the Theory of Rings with Minimum Conditions," Science Reports of the Tokyo Kyoiku Daigoku Section A, Vol. 6, 1958, pp. 83-142.

[4] Y. Wang and Y. L. Ren, "Morita Context Ring with a Pair of Zero Homomorphism I," Journal of Jilin University (Science Edition), Vol. 44, No. 3, 2006, pp. 318-324.

[5] Y. Wang and Y. L. Ren, "Morita Context ring with a Pair of Zero Homomorphism II," Journal of Mathematical Research and Exposition, Vol. 27, No. 4, 2007, pp. 687692.

[6] H. Ebrahim, “A Note on p.q.-Baer Modules," New York Journal of Mathematics, Vol. 14, 2008, pp. 403-410.

[7] T. W. Hungerford, "Algebra," Springer-Verlag, New York, 1980.

[8] S. Montgomery, "Fixed Rings of Finite Automorphism Groups of Associative Rings,” Springer-Verlag Berlin Heidelberg, 1980.

[9] T. Y. Lam, "Lectures on Modules and Rings," SpringerVerlag, New York, 1999. doi:10.1007/978-1-4612-0525-8

[10] S. T. Rizvi and S. R. Cosmin, "Baer and Quasi Baer Modules," Communications in Algebra, Vol. 32, No. 1, 2004, pp. 103-123. doi:10.1081/AGB-120027854 